ACTUALIZACIÓN EN

\title{
La adherencia terapéutica y las enfermedades crónicas. Un problema clínico
}

[Therapeutic adherence and chronic diseases. A clinical problem]

\section{Virginia Díaz A. ${ }^{1}$}

${ }^{1}$ Departamento de Neumología, Hospital del Niño Dr. José Renán Esquivel. Panamá, República de Panamá. Correspondencia Dra.Virginia Díaz A. / Correo electrónico:vedadiaz@gmail.com

Recibido: 20 de julio del 2021

Aceptado: 01 de septiembre del 2021

Publicado: 30 de diciembre de 2021

Palabras clave: enfermedades crónicas, adherencia terapéutica, asma bronquial

Keywords: chronic diseases, therapeutic adherence, bronchial asthma

Reproducción: Artículo de acceso libre para uso personal e individual. Sujeto a derecho de reproducción para otros usos.

Aspectos bioéticos: El autor declara no tener conflictos de interés, y que la información cruda no podrá ser compartida.

Financiamiento: El autor declara no tener fuentes externas de financiamiento asociados a este trabajo.

DOI: $10.37980 /$ im.journal. rspp.20211786

\section{Resumen}

La aceptación de padecer una enfermedad crónica y aprender a convivir con ésta puede ser un proceso lento para el paciente y su familia. Los niños y los adolescentes con una enfermedad crónica se enfrentan a un desafío que requiere afrontar la responsabilidad y exigencias de la necesidad de un tratamiento diario a largo plazo que muchas veces requiere adaptar sus actividades y su vida para cumplir con el mismo. El objetivo del tratamiento en las enfermedades crónicas es minimizar los síntomas, alcanzar el control o modificar la evolución natural para lograr una calidad de vida adecuada. Adherencia significa la aceptación de un rol activo en el propio cuidado de la salud, pero que debe comprender la colaboración entre el paciente, su familia y el equipo de salud. Esta colaboración debe incluir confianza, términos como negociación, toma de decisiones en forma conjunta y comunicación para determinar el tratamiento adecuado de la enfermedad. La adherencia al tratamiento es un problema y constituye un reto y una preocupación para los médicos que tratan a pacientes con enfermedades crónicas porque la falta de adherencia genera empeoramiento de la enfermedad, aumento del número de hospitalizaciones, altos costos para el paciente, la familia y el sistema de salud.

\section{Abstract}

Accepting to suffer from a chronic disease and learning to live with this disease can be a slow process for the patient and their family. Children and adolescents with a chronic disease face a challenge that requires facing the responsibility and demands of the need for long-term daily treatment that often requires adapting their activities and their lives to comply with it. The goal of treatment in chronic diseases is to minimize symptoms, achieve control, or modify the natural history to achieve an adequate quality of life. Adherence means the acceptance of an active role in one's own health care but that must include collaboration between the patient, his family and the health team. This collaboration should include trust, terms such as negotiation, joint decision-making, and communication to determine the appropriate treatment for the disease. Adherence to treatment is a problem and constitutes a challenge and concern for physicians who treat patients with chronic diseases because lack of adherence leads to a worsening of the disease, an increase in the number of hospitalizations, high costs for the patient, the family and the health system. 


\section{Introducción}

La OMS establece que la adherencia terapéutica es un fenómeno multidimensional determinado por la acción recíproca de cinco dimensiones, de los cuales los factores relacionados con el paciente son solo un determinante.

La adherencia se define como la medida en que la conducta de una persona coincide con los consejos o recomendaciones de salud. La definición de adherencia terapéutica de la Organización Mundial de la Salud (OMS) es

"El grado en que el comportamiento de una persona - tomar el medicamento, seguir un régimen alimentario y ejecutar cambios del modo de vida - se corresponde con las recomendaciones acordadas con un prestador de asistencia sanitaria." ${ }^{2}$

Para que haya adherencia al tratamiento es necesario que ocurran los siguientes eventos: ${ }^{3}$

a) Aceptación convenida del tratamiento, entre el paciente y su médico, entendida como la relación de colaboración establecida entre médico y paciente, con vistas a elaborar la estrategia comportamental que garantiza el cumplimiento y la aceptación de ésta por ambos.

b) Cumplimiento del tratamiento como la medida en que la persona ejecuta todas las prescripciones médicas indicadas por el terapeuta.

c) Participación en el cumplimiento, entendido como el grado de participación del paciente en la búsqueda de estrategias para garantizar el cumplimiento.

d) Carácter voluntario de las acciones para el cumplimiento, entendido como la medida en que la persona participa en la ejecución de las acciones realizando los esfuerzos necesarios para garantizar el cumplimiento de las prescripciones.

Los determinantes relacionados con la adherencia pueden agruparse en factores socioeconómicos, los relacionados con el equipo o el sistema de asistencia sanitaria, con la enfermedad, con el tratamiento y con el paciente. ${ }^{1}$ Deben considerarse todos estos factores para ofrecer alternativas o acciones encaminadas a superar los problemas de la adherencia.

\section{Factores socioeconómicos}

La pobreza, el analfabetismo, el desempleo, la falta de redes de apoyo, la lejanía de los centros de tratamiento, el costo de la medicación, la cultura y las creencias populares acerca de la enfermedad y el tratamiento pueden tener un efecto considerable sobre la adherencia., ${ }^{1,4}$

Factores relacionados con el equipo o el sistema de asis- tencia sanitaria

Una buena relación con el equipo tratante puede mejorar la adherencia. Hay muchos factores que ejercen un efecto negativo como los sistemas deficientes de distribución de medicamentos, falta de adiestramiento del personal sanitario en el control de las enfermedades crónicas y falta de conocimiento sobre la adherencia y las intervenciones efectivas para mejorarla, profesionales recargados de trabajo, poca capacidad del sistema para educar a los pacientes y proporcionar seguimiento, incapacidad para establecer el apoyo de la comunidad y la capacidad de autocuidado. ${ }^{1,4}$

\section{Factores relacionados con la enfermedad}

Algunos determinantes son la gravedad y la velocidad de progresión de esta, el grado de discapacidad física, psicológica, social, la disponibilidad de tratamientos efectivos. Así como la comorbilidad con trastornos emocionales o consumo de sustancias. ${ }^{1,4}$

\section{Factores relacionados con el tratamiento}

Los más importantes están relacionados con la complejidad del régimen médico, la duración del tratamiento, los fracasos de tratamientos anteriores, los cambios frecuentes en el tratamiento, la inminencia de los efectos beneficiosos, los efectos adversos y la disponibilidad de apoyo médico para tratarlos. ${ }^{1,4}$

\section{Factores relacionados con el paciente}

Representan los recursos, el conocimiento, las actitudes, las creencias, los temores, la presencia de procesos de duelo, las percepciones y las expectativas del paciente y su familia. ${ }^{1}$

Se ha reportado que aproximadamente el $50 \%$ de los pacientes con enfermedades crónicas no se adhieren adecuadamente al tratamiento. ${ }^{2}$

La no adherencia puede ser intencional o no intencional. La primera cuando ocurre por una decisión consciente, por ejemplo, por preocupaciones o falta de confianza en los medicamentos, cuando los padres equilibran los efectos positivos y negativos de la medicación prescrita y cambian deliberadamente de la indicación del médico y en caso específico de los adolescentes por problemas psicosociales. La no adherencia no intencional puede ocurrir porque no se sigue el tratamiento por desconocimiento, técnica de administración inadecuada u olvido, ausencia de las rutinas diarias en la toma de los medicamentos, negativa del niño a tomar la medicación, aceptación del nivel actual de adherencia como el mejor posible y por transferencia de responsabilidad excesiva a los adolescentes..$^{5-7}$

La falta de adherencia es en gran parte responsable de las diferencias en la eficacia de los medicamentos observadas en la práctica clínica versus la eficacia reportada en los ensayos clínicos. ${ }^{8}$ 
Es necesario estar familiarizados con las etapas del desarrollo para poder diseñar e implementar diferentes estrategias e intervenciones.

A medida que el niño va creciendo puede ir tomando gradualmente mayor responsabilidad en la toma de medicación o en el control de variables médicas. Las medidas que vaya adoptando para controlar en forma cada vez más independiente su enfermedad, se relacionan con el desarrollo cognitivo, la adquisición de autonomía y la menor dependencia de los adultos.

El diagnóstico de una enfermedad crónica en los lactantes puede interferir en el vínculo madre-hijo, generando sentimientos de culpa, empeorando la comprensión y aceptación del problema de salud. ${ }^{1}$

En este grupo las intervenciones del equipo de salud deben centrarse en el apoyo y educación hacia los cuidadores y es necesario comunicar los conocimientos acerca del origen del trastorno y anticipar los cambios que se van a ir generando durante el desarrollo del niño.

Los cuidadores deben ser entrenados para adquirir habilidades en el manejo de la medicación y de los síntomas para una mejor crianza del niño. Por eso es necesario tener en cuenta las rutinas del niño y la familia, el tipo de alimentación, los gustos, intereses y la capacidad de los adultos para aceptar indicaciones.

Durante la etapa preescolar los niños necesitan mantener esquemas regulares y rutinas. En esta etapa es útil incluir juegos para ayudarlos a llegar a transformar lo penoso en soportable. Cuando reciben medicación desde etapas tempranas, ésta pasa a ser parte de su vida y suele ser mejor aceptada. ${ }^{1}$

Para una mejor adherencia al tratamiento es necesario evaluar el rol de los adultos o cuidadores, respecto de la forma y modo de administrar la medicación, conocimientos acerca de la evolución de la enfermedad y consecuencias de una mala adherencia. Se aconseja que al menos dos personas de la familia conozcan la medicación o la rutina del tratamiento para que estén disponibles y organizadas a tal efecto. Es necesario que los niños y sus padres comprendan que el tratamiento no es un área de negociación de su cumplimiento.

La información que reciban el niño y sus padres deberá estar orientada a entender los beneficios a corto y largo plazo de una correcta adherencia a los tratamientos.

El período de 6 a 12 años es una etapa de avances en cuanto a la socialización y la objetivación del pensamiento. En esta etapa es fundamental la integración a la escuela, a grupos de pares para la adquisición y consolidación de habilidades sociales y el desarrollo de la expresión de sentimientos. Nece- sitan conocer aspectos concretos de la enfermedad y del tratamiento médico que deben seguir, y la información deberá estar orientada a que colabore según su nivel de desarrollo. ${ }^{1}$

En lo posible es importante que la escuela esté informada y actualizada del estado de salud del niño y sus necesidades.

La adolescencia se determina por factores biológicos, sociales y psicológicos. Es un momento de gran vulnerabilidad psicológica, en donde es prioritario considerar al adolescente en forma integral. En esta etapa ya pueden comprender y asimilar los conocimientos relacionados a su enfermedad, las consecuencias de una deficiente adherencia, pero les cuesta entender la cronicidad del cuadro $y$, muy especialmente, mantener un adecuado cumplimiento de las pautas de tratamiento. ${ }^{1}$

A esta edad pueden aparecer sentimientos conflictivos ligados a la enfermedad y los jóvenes pueden reaccionar suspendiendo la medicación para sentirse "normales". Sus principales temores son los de la mayoría de su grupo etario: pérdida del control, ser diferentes al resto, ser discriminados o ignorados por sus pares.

En una etapa en la que el aspecto físico cobra especial interés, los cambios en la imagen corporal, caída del cabeIlo, o incremento de sus discapacidades físicas producidos por el tratamiento, pueden ser grandes obstáculos para la adherencia y pueden generar pérdida de interés por la vida.

La fluctuación de las emociones es habitual, pasando de la desesperación y pesimismo a la euforia y a sentimientos de omnipotencia. Una mala relación con las reglas, una actitud de base desafiante, poca conciencia de enfermedad y ausencia de síntomas físicos, e incompatibilidad entre el esquema de tratamiento y el estilo de vida del adolescente pueden ser obstáculos importantes para lograr la adherencia en esta etapa.

Es frecuente que los cuidadores adopten una actitud sobreprotectora para con el paciente. Esta actitud suele generar demoras en la adquisición de la independencia. Los adolescentes con enfermedades crónicas tienen un elevado riesgo de padecer aislamiento social y retracción en los vínculos extra familiares para evitar sentirse expuestos.

En esta etapa pueden ser beneficiosos los grupos de apoyo con otros pacientes adolescentes, o conectarse en un modo más informal con otros jóvenes que padecen una problemática similar. Es importante promover la participación activa del adolescente en su tratamiento que sirva para que pueda asumir responsabilidades y tomar decisiones; pero, aún esta participación, requiere siempre de la supervisión de un adulto. 
Es importante que el paciente comprenda las consecuencias de nocumplirconeltratamiento, entendiendoque, sibiennosepodrá curar, el tratamiento le permitirá una mejor calidad de vida. La adherencia a la medicación para el asma varía según grupos de edad y con el tipo de medida utilizada. Se han obtenidos datos de niveles de adherencia, medidos objetivamente, del 49 al $71 \%$ en niños y adolescentes asmáticos, aunque la adherencia autoinformada suele ser mayor. ${ }^{5-7}$

En una revisión sistemática de estudios cualitativos sobre la adherencia al tratamiento en niños con diversas afecciones a largo plazo, incluido el asma, los cuidadores informaron la importancia de equilibrar varias preocupaciones, incluida sus creencias sobre una afección y su tratamiento, resistencia infantil, preservar las relaciones familiares y promover una "vida normal" para su familia. ${ }^{9}$

Varias encuestas mostraron que el conocimiento sobre la medicación para el asma es subóptimo tanto en padres como en adolescentes. ${ }^{10,11} \mathrm{El}$ conocimiento insuficiente sobre el asma y sus tratamientos, así como las preocupaciones y el miedo a los efectos secundarios por el uso diario de corticoides inhalados se han relacionado a la falta de adherencia y un menor control de la enfermedad ${ }^{10-12}$ apoyando la importancia de mejorar la educación del paciente y sus padres. También se ha encontrado que los pacientes y sus padres están predispuestos a sobrestimar el control del asma y esto influye en que la adherencia a la medicación en niños y adolescentes asmáticos sea subóptima. ${ }^{13}$

Las razones de la no adherencia pueden diferir entre niños y adolescentes asmáticos. Para los niños más pequeños, los padres suelen estar más involucrados en la toma de medicamentos, mientras que los adolescentes son responsables de tomar sus medicamentos. ${ }^{6}$ En un estudio de adherencia en los pacientes asmáticos los padres de niños entre 18 meses y 7 años informaron olvido y la reacción de su hijo a recibir medicación como las razones más comunes para la falta de adherencia. ${ }^{14}$ En un estudio en adolescentes aproximadamente la mitad de los casos de incumplimiento ocurrió en presencia de amigos. ${ }^{15}$ El olvido también se ha relacionado con la falta de adherencia en los adolescentes. ${ }^{16,17}$

Psicosocialmente, el adolescente debe equilibrar su deseo para la autonomía, la comunicación y el conflicto familiar, las relaciones con los compañeros y las demandas académicas y vocacionales dentro del contexto del manejo de la enfermedad.

Los adolescentes con enfermedades crónicas tienen más posibilidades de presentar comportamientos de riesgo en comparación con sus pares.$^{18}$ Los adolescentes con asma informan niveles más altos de ansiedad social, sentimientos depresivos, autoestima comprometida y suicidio que sus pares no asmáticos lo que puede disminuir la adherencia al tratamiento. ${ }^{19-24}$
Aproximadamente un tercio de los adolescentes diagnosticados con asma puede sufrir un trastorno de ansiedad o pánico. El trastorno de ansiedad social también es más prevalente entre los adolescentes con asma, tal vez debido a su miedo a la evaluación negativa de sus compañeros y al aumento de la incomodidad en los entornos sociales. ${ }^{22,25}$

Entre el $20 \%$ y el $50 \%$ de los adolescentes diagnosticados con asma informan síntomas depresivos significativos. ${ }^{26} \mathrm{Un}$ metaanálisis informó una tasa de prevalencia de depresión del $27 \%$, que es más del doble que la de los adolescentes sin asma. ${ }^{27}$ Los síntomas depresivos, en los adolescentes asmáticos y en la población adolescente general, parecen estar especialmente relacionados con otras comorbilidades conductuales, como las conductas de riesgo, el tabaquismo y el abuso de sustancias. ${ }^{19,21}$

Las interacciones exitosas con los compañeros durante la adolescencia afectan la formación de la identidad, la auto- imagen, el ajuste psicológico y la adherencia al tratamiento. ${ }^{26,27}$ Los sentimientos de vergüenza por el asma se han relacionado con no llevar un inhalador o tener menos probabilidades de usar medicamentos para el asma frente a compañeros. Los adolescentes también pueden optar por no usar medicamentos por temor a ser "interrogados" sobre su enfermedad y sus medicamentos frente a otros. ${ }^{28}$

Las consecuencias de una adherencia deficiente para los adolescentes con asma incluyen la falta de control y una mayor morbilidad por asma. ${ }^{29,30}$ Además, el asma mal controlada disminuye la calidad de vida relacionada con la salud de los niños y adolescentes y es responsable del aumento de las ausencias escolares, las visitas a la sala de emergencias, las visitas al consultorio, un mayor consumo de agonistas $\beta 2$ de acción corta y cambios innecesarios al alza en los escalones terapéuticos y la atención hospitalaria a un costo de más de mil millones de dólares por año. ${ }^{31-33}$

Las barreras para la adherencia de los adolescentes incluyen actitudes negativas hacia los proveedores de atención médica, ${ }^{34}$ reticencia a tomar medicamentos en presencia de pares y negación de las consecuencias de la falta de adherencia..$^{35}$ Los pacientes adolescentes diagnosticados con asma informaron frecuentes barreras para la adherencia, que incluyen: el tratamiento y las actividades diarias interfieren entre sí, olvidos, dificultades psicosociales y querer parecer normales a sus compañeros. Los padres de adolescentes informaron que el olvido, el desagrado por el sabor de la medicación, la conducta de oposición y el tratamiento y las actividades diarias interfieren entre sí como barreras frecuentes, también se ha encontrado que la información errónea sobre los medicamentos para el asma y suposiciones incorrectas como "superar el asma " contribuyen a la falta de adherencia. ${ }^{36,37}$ 
Los métodos para medir la adherencia al tratamiento del asma pueden dividirse en directos $u$ objetivos e indirectos o subjetivos. Los métodos directos son el uso de dispositivos electrónicos y la determinación del nivel de fármacos. Los métodos indirectos son juicio clínico, cuestionarios autorreferidos, base de datos farmacéuticos, conteo de dosis y peso de los recipientes. ${ }^{6,32,38-41}$ Estos métodos difieren en precisión, esfuerzo y costo.

Las mediciones subjetivas tienden a sobreestimar el nivel de adherencia en comparación con las mediciones objetivas. ${ }^{6,14,42,43}$

Los métodos indirectos son sencillos y baratos. No son objetivos, por lo que identifican principalmente a una parte de los pacientes no adherentes al tratamiento. La mayoría no cuantifican, pero proporcionan una puntuación en la vida real.

Al evaluar la adherencia a la medicación, el autoinforme o cuestionario auto referido es el método más rentable, es fácil de utilizar y demanda poco tiempo y también permite la recopilación de información. sobre la actitud y las creencias de los pacientes. ${ }^{44}$ Tiene la desventaja de sobreestimar la adherencia terapéutica en comparación con los métodos objetivos. $39,45^{30}$

Todos los cuestionarios autoreferidos, excepto el Test de Adhesión a los Inhaladores ${ }^{46}$ están diseñados o son extrapolados de otras enfermedades, lo que limita en cierta manera su utilidad en la práctica clínica habitual. ${ }^{39} \mathrm{El}$ TAI identifica la inadecuada adherencia, su graduación, permite valorar el tipo de incumplimiento (errático, deliberado, inconsciente) e incorpora además la valoración de la técnica inhalatoria, pero está validada su aplicación a partir de los 18 años. ${ }^{46}$

Las bases de datos farmacéuticos permiten calcular la adherencia comparando la prescripción realizada a través de la receta electrónica con la medicación dispensada en las farmacias en un tiempo determinado. Su principal inconveniente es que no garantiza que el sujeto tome o inhale la medicación. ${ }^{39,45}$

Los métodos directos se basan en la cuantificación de un fármaco, de sus metabolitos o de una sustancia trazadora en algún fluido biológico.

Son objetivos, específicos, exactos, fiables, caros, complejos y están limitados a ciertos fármacos. Son útiles en los ensayos clínicos y permiten, además de valorar el cumplimiento, optimizar rangos terapéuticos en determinadas pautas de tratamiento. Es evidente que son métodos perfectos para valorar el cumplimiento; sin embargo, pueden sobreestimarlo y resultar incómodos e invasivos. ${ }^{39}$

Los dispositivos electrónicos son instrumentos que pueden adaptarse a los inhaladores y permiten saber el número de dosis suministradas y la fecha y hora de su aplicación, y en algunos se pueden proporcionar mensajes de recuerdo sobre la toma de la medicación o la técnica inhalatoria, con el objetivo de incrementar la adherencia terapéutica. ${ }^{47}$ Los datos se pueden descargar en un ordenador y ser analizados posteriormente. Son caros y pueden presentar problemas técnicos hasta en un $20 \%$ en cuanto al almacenaje de la información, la transmisión de datos y la carga de batería. ${ }^{48-50}$

Se ha creado y validado una nueva herramienta electrónica, INhaler Compliance Assessment (INCA) ${ }^{51}$ que, además permite evaluar la técnica inhalatoria y la conjuga con el tiempo de uso. Se basa en la grabación del audio durante el uso del dispositivo inhalador, pudiendo detectar entre otros errores técnicos un flujo inspiratorio bajo, una baja duración de la inspiración y múltiples inhalaciones para una misma dosis. ${ }^{52-54}$

La determinación de niveles séricos como el cortisol pueden identificar si la medicación, corticoides inhalados, se toma regularmente, al producir una supresión endógena completa o parcial de los niveles de cortisol. También se cuenta con la medición sérica de teofilina, aunque este medicamento solo es utilizado por una pequeña cantidad de pacientes. ${ }^{39}$

La evaluación de la adherencia es un proceso continuo y crucial para manejo del asma, por lo tanto, mejorar la adherencia terapéutica es muy importante para alcanzar la meta del tratamiento del asma que es lograr el control de esta enfermedad. Para mejorar la adherencia terapéutica debemos identificar, cuantificar, valorar y clasificar el o los problemas que determinan la falta de adherencia y dar seguimiento para proporcionar las medidas específicas e individualizadas en cada paciente.

Para mejorar la adherencia terapéutica se pueden intentar diferentes intervenciones que deben ser dirigidas a la causa identificada para la no adherencia. (Tabla 1)

Como primer paso, es necesario evaluar el conocimiento del paciente y de la familia con relación a la enfermedad y su tratamiento. Se debe enfatizar el rol de apoyo de la familia en relación con el tratamiento de su hijo para brindar la supervisión adecuada, ayudando a anticipar las dificultades que puedan interferir con la adherencia.

La educación es parte fundamental del tratamiento de la enfermedad ya que proporciona al paciente los conocimientos y las habilidades necesarias para mejorar la adherencia terapéutica y promover el autocuidado y mejorar el control de la enfermedad. Un plan de acción escrito ha mostrado su eficacia al producir un incremento en la adhesión y prevenir las consecuencias del incumplimiento terapéutico. ${ }^{55-57}$ Debe ser individualizado y revisarse periódicamente, ya que se ha constatado que la mejoría del cumplimiento disminuye con el tiempo..$^{55,58}$ 
Tabla 1. Factores relacionados con la adherencia terapéutica y recomendaciones para mejorar la adherencia.

\begin{tabular}{|c|c|}
\hline $\begin{array}{l}\text { Factores relacionados con el } \\
\text { medicamento }\end{array}$ & Intervenciones para mejorar la adherencia \\
\hline Dificultades con el inhalador & $\begin{array}{ll}\text { - } & \text { Identificar el inhalador apropiado para el paciente. } \\
\text { - } & \text { Enseñar su uso y hacer que el paciente reproduzca la técnica }\end{array}$ \\
\hline Régimen difícil o múltiples fármacos & - Simplificar el régimen según las preferencias del paciente \\
\hline Miedo sobre efectos secundarios & $\begin{array}{l}\text { - } \quad \text { Entrevistas motivacionales para determinar pros y contras y reducir ambivalencia. } \\
\text { - Considerar referir a grupos de apoyo }\end{array}$ \\
\hline Costo de la medicación & $\begin{array}{l}\text { - Seleccionar el medicamento menos costoso, referir a farmacias con planes de } \\
\text { des-cuento o programas de asistencia farmacéutica }\end{array}$ \\
\hline Desacuerdo con la medicación & $\begin{array}{l}\text { - } \quad \text { Reducir exposición a alérgenos o irritantes para reducir síntomas o dosis de medica-ción. } \\
\text { - } \quad \text { Entrevistas motivacionales para determinar pros y contras y reducir ambiva-lencia }\end{array}$ \\
\hline $\begin{array}{l}\text { Farmacias lejanas } \\
\text { Factores relacionados al paciente }\end{array}$ & - Identificar capacidad de recibir la medicación por correo o por entrega a domicilio \\
\hline $\begin{array}{l}\text { Falta de comprensión de las } \\
\text { indicaciones }\end{array}$ & $\begin{array}{l}\text { - } \quad \text { Proporcionar instrucciones. } \\
\text { - } \quad \text { Valorar alfabetismo. Si es bajo, adaptar estrategias de edu-cación. } \\
\text { - } \quad \text { Explicar fisiopatología y relacionar con el tratamiento, así como con las consecuencias de } \\
\text { no cumplirlo. } \\
\text { - } \quad \text { Enseñar la técnica inhalatoria }\end{array}$ \\
\hline Insatisfacción con el personal de salud & $\begin{array}{l}\text { - } \quad \text { Hacer que el paciente hable con la persona encargada. } \\
\text { - } \quad \text { Puede ser necesario que el paciente sea visto por otro profesional si la relación no mejora }\end{array}$ \\
\hline $\begin{array}{l}\text { Miedos o preocupaciones no } \\
\text { manifestadas }\end{array}$ & $\begin{array}{l}\text { - } \quad \text { Identificar preocupaciones y dirigir cada una. } \\
\text { - } \quad \text { Determinar si tienen fundamento o no. } \\
\text { - } \quad \text { Considerar referir a un grupo de apoyo. }\end{array}$ \\
\hline Expectativas inapropiadas & $\begin{array}{l}\text { - } \quad \text { Aclarar expectativas desde una perspectiva médica. } \\
\text { - Si el paciente espera una mejoría mayor o más rápida, intentar reestablecer las } \\
\text { expectativas. } \\
\text { - } \quad \text { Revalorar el rol de la exposición a alérgenos/irritantes }\end{array}$ \\
\hline $\begin{array}{l}\text { Poca supervisión, entrenamiento o } \\
\text { seguimiento }\end{array}$ & $\begin{array}{ll}\text { - } & \text { Alentar a supervisar a niños. } \\
\text { - } & \text { Revisar el uso de la medicación en la consul-ta. } \\
\text { - } & \text { Programar apropiadamente visitas de seguimiento }\end{array}$ \\
\hline $\begin{array}{l}\text { Disgusto por la enfermedad o su } \\
\text { tratamiento }\end{array}$ & $\begin{array}{l}\text { - Identificar la causa de disgusto. } \\
\text { - } \quad \text { Aclarar que el tratamiento puede mejorar la enfermedad. } \\
\text { - } \quad \text { vvaluar ambivalencia sobre el tratamiento y revisar posibles alternativas. }\end{array}$ \\
\hline Subestimar la gravedad del asma & $\begin{array}{l}\text { - Relacionar síntomas con la función pulmonar o realizar pruebas de ejercicio para } \\
\text { demostrar la gravedad de la condición. }\end{array}$ \\
\hline Factores culturales & $\begin{array}{l}\text { Entender que varias culturas tienen conceptos diferentes de desarrollo del asma, } \\
\text { fac-tores que producen exacerbaciones y elecciones de tratamiento. } \\
\text { - Aprovechar a todos los trabajadores de salud para aclarar estos factores. }\end{array}$ \\
\hline Preocupaciones sobre estigmatización & $\begin{array}{l}\text { - Evaluar la reacción del paciente ante el diagnóstico. } \\
\text { - } \quad \text { Comprender las preocupaciones del paciente y referir a grupos de apoyo si éstas } \\
\text { persisten. }\end{array}$ \\
\hline Olvido & $\begin{array}{l}\text { - } \quad \text { Detectar el problema u otras razones por las que olvida tomar la medicación. } \\
\text { - } \quad \text { Rensi-derar asociar el uso de la medicación con actividades habituales. } \\
\text { Rento para valorar la necesidad actual del mismo. }\end{array}$ \\
\hline Actitud hacia la enfermedad & $\begin{array}{l}\text { - Evaluar la creencia del paciente sobre el asma y su tratamiento. } \\
\text { - En pacientes que cuestionan el diagnóstico o la eficacia del tratamiento, considerar } \\
\text { suspender el trata-miento y monitorizar la función pulmonar en el domicilio. }\end{array}$ \\
\hline Factores religiosos & $\begin{array}{l}\text { Valorar cómo la creencia religiosa del paciente puede afectar la actitud sobre el } \\
\text { diagnóstico y el tratamiento. Hablar con su líder religioso puede ser un soporte para el } \\
\text { paciente. }\end{array}$ \\
\hline
\end{tabular}

Modificado de Weinstein AG. Asthma adherence management for the clinician. J Allergy Clin Immunol Pract. 2013;1:123-8 ${ }^{45}$ 
Tabla 2. Educación en asma pediátrica. Metaanálisis publicados desde 2000

\begin{tabular}{|c|c|c|c|}
\hline Autor & Estudio & Objetivos & Resultado principal \\
\hline Haby & 8 estudios en niños & $\begin{array}{l}\text { Impacto de la educación en las } \\
\text { visitas a urgencias y después de } \\
\text { ellas }\end{array}$ & $\begin{array}{l}\text { Sin modificación en las visitas a } \\
\text { urgencias ni después }\end{array}$ \\
\hline Guevara & $\begin{array}{l}32 \text { estudios en niños / } \\
\text { adolescentes }\end{array}$ & $\begin{array}{l}\text { Flujo espiratorio pico basado en } \\
\text { un plan de acción }\end{array}$ & $\begin{array}{l}\text { Disminución de las visitas a urgencias } \\
\text { Disminución de síntomas y mejoría en } \\
\text { la función pulmonar }\end{array}$ \\
\hline Toelle & $\begin{array}{l}7 \text { estudios en niños / } \\
\text { adolescentes }\end{array}$ & $\begin{array}{l}\text { Flujo espiratorio de los planes } \\
\text { de acción individuales }\end{array}$ & Sin mejoría \\
\hline Smith & $\begin{array}{l}57 \text { estudios ( } 21 \text { adultos, } \\
35 \text { niños) en asma } \\
\text { grave }\end{array}$ & $\begin{array}{l}\text { Impacto de planes de acción } \\
\text { individualizados (síntomas vs } \\
\text { flujo espiratorio pico) }\end{array}$ & $\begin{array}{l}\text { Reducción baja de hospitalizaciones en } \\
\text { adultos y niños. Mejoría de síntomas en } \\
\text { niños. }\end{array}$ \\
\hline Bhogal & $\begin{array}{l}4 \text { estudios en niños / } \\
\text { adolescentes }\end{array}$ & $\begin{array}{l}\text { Impacto de planes de acción } \\
\text { individualizados (síntomas vs } \\
\text { flujo espiratorio pico) }\end{array}$ & $\begin{array}{l}\text { Los síntomas identificados según los } \\
\text { planes de acción fueron más eficientes } \\
\text { que el flujo espiratorio pico en la } \\
\text { disminución de las visitas a urgencias. }\end{array}$ \\
\hline Bailey & $\begin{array}{l}4 \text { estudios en niños / } \\
\text { adultos }\end{array}$ & $\begin{array}{l}\text { Cultural vs enfoque educativo } \\
\text { tradicional }\end{array}$ & $\begin{array}{l}\text { Mejoró el conocimiento. } \\
\text { Mejoró el cuestionario de calidad de } \\
\text { vida. }\end{array}$ \\
\hline Coffman & $\begin{array}{l}37 \text { estudios en niños 2- } \\
17 \text { años }\end{array}$ & $\begin{array}{l}\text { Atención sanitaria en } \\
\text { situaciones de emergencia }\end{array}$ & $\begin{array}{l}\text { Disminuyeron las tasas de } \\
\text { hospitalización y visitas a urgencias. Sin } \\
\text { modificación en las visitas } \\
\text { programadas. }\end{array}$ \\
\hline Server & Niños & $\begin{array}{l}\text { Comparar estrategias } \\
\text { educativas dirigidas hacia el } \\
\text { paciente vs la enfermedad }\end{array}$ & $\begin{array}{l}\text { Estrategias dirigidas hacia el paciente } \\
\text { mejoraron el control del asma. }\end{array}$ \\
\hline
\end{tabular}

Modificado de Melero Moreno C, Rodríguez Meleán N, Díaz Campos R. Rev Asma. 2017;2(1):13-22 39

Siempre debe explicarse por qué y cómo se debe usar la medicación inhalada, simplificar el tratamiento y tener en cuenta que no todos los inhaladores son adecuados para un enfermo y sus preferencias. ${ }^{59}$ Se debe simplificar el plan de tratamiento y discutir las expectativas del paciente y la familia en relación a los resultados esperados. ${ }^{60}$ Se presentan los estudios y resultados de metaanálisis de intervenciones educativas y sus resultados publicados desde el año 2000 (Tabla 2).

Los padres suelen estar más involucrados en la toma de medicamentos cuando se trata de niños pequeños, mientras que los padres de adolescentes piensan que ellos son responsables de tomar sus medicamentos; sin embargo, la transferencia de responsabilidad excesiva para el adolescente apoya la importancia de la educación para el paciente y sus padres para lograr una adherencia óptima. ${ }^{1,6,13}$

Las intervenciones de adherencia para adolescentes con asma también han utilizado la tecnología, incluidos los mensajes de texto, Internet y los medios de comunicación social. ${ }^{60}$ Son herramientas creadas en la web que pueden involucrar planes educativos, hojas de recogida de datos clínicos periódicos, planes de autocuidados y planes de acción. Los diferentes estudios que han evaluado esta opción han comunicado resultados variables en relación con una mejoría en síntomas, exacerbaciones y calidad de vida. Se ha comprobado que su utilización disminuye con el número de accesos a la herramienta. ${ }^{39,61-63}$ Un estudio piloto encontró mejoras en los síntomas del asma al mes y a los tres meses de seguimiento en un grupo de intervención que recibió mensajes de texto personalizados. ${ }^{64}$

Los adolescentes son más susceptibles a la influencia social, la presión y opinión de sus compañeros. Se ha descrito que un programa de autocontrol del asma dirigido por pares fue bien recibido por los adolescentes ${ }^{65}$ lo que sugiere que los adolescentes pueden estar más dispuestos a aprender de líderes que se perciben como similares a ellos. Por esta razón, se podrían utilizar las redes sociales y es probable que la tecnología móvil sea una estrategia adecuada en adolescentes asmáticos.

Podemos resumir el tipo de estrategias para mejorar la adherencia terapéutica según los problemas identificados así:

Para los pacientes con una adherencia errática, las medidas a incorporar incluyen simplificar el tratamiento, asociar la medicación con alguna actividad habitual como lavarse los dientes o el uso de dispositivos que recuerden la toma de 
la medicación como aplicaciones para los dispositivos móviles, dispositivos electrónicos con capacidad de enviar mensajes, llamadas telefónicas de recuerdo o interactivas, SMS y otras. ${ }^{39,45,55}$

Si se trata de pacientes con una inadecuada adherencia intencional, una posible intervención es realizar entrevistas motivacionales para identificar, conflictos, preocupaciones, creencias y temores. ${ }^{39,45}$ Los estudios de intervención en la adherencia demuestran la importancia de centrarse en las creencias del adolescente relacionadas con el cambio de comportamiento de salud. ${ }^{66,67}$ Las discusiones deben centrarse en cómo los padres y los adolescentes pueden compartir la responsabilidad, incluido un programa para reducir gradualmente la participación de los padres en el cuidado diario del asma del adolescente. Un plan estructurado puede reducir el conflicto entre padres e hijos adolescentes sobre el cuidado del asma en el hogar.

Las barreras para la adherencia relacionadas con la práctica médica y el sistema de salud son importantes y enfatizan la responsabilidad del médico de mejorar la práctica diaria de la medicina para aumentar la adherencia y, por lo tanto, los resultados de los pacientes. La alfabetización en salud con la transmisión adecuada de información sobre la enfermedad y los medicamentos, mantener una comunicación adecuada, simplificar el régimen de tratamiento en cuanto a la cantidad de medicamentos y la frecuencia de administración y tener en cuenta la rutina del paciente en relación a los horarios al recetar los medicamentos son acciones que podemos implementar para mejorar la adherencia terapéutica. ${ }^{4}$

\section{Conclusiones}

La adherencia terapéutica es importante para un buen control de los síntomas de los pacientes con enfermedades crónicas. La adherencia al tratamiento es un proceso complejo que requiere de estrategias de educación sanitaria a la población y a los prestadores de salud.

En la práctica clínica habitual se necesita reconocer, cuantificar y seguir la evolución en el tiempo de la adherencia terapéutica y centralizar los esfuerzos en intervenciones multidisciplinarias específicas e individualizadas que eliminen las barreras en la adherencia terapéutica.

Se debe enfatizar el rol de apoyo de la familia para brindar la supervisión adecuada, ayudando a anticipar las dificultades que puedan interferir con la adherencia.

No existe ningún procedimiento de medición de adherencia ideal o sin limitaciones, por lo que probablemente lo mejor sería la utilización combinada de los métodos disponibles.

Todos los proveedores del sistema de atención de la salud debemos participar en fomentar la adherencia terapéutica. Las enfermeras, los farmacéuticos y los médicos que tene- mos contacto con los pacientes podemos hacer contribuciones importantes. Los médicos tenemos la responsabilidad de minimizar las barreras al incumplimiento terapéutico cambiando nuestro propio comportamiento.

\section{Referencias}

1. Bordato A, Nielsen V, Norton E. Adherencia al tratamiento en niños y adolescentes. Medicina Infantil. 2017; 34 (2):155-159

2. Organización Mundial de la Salud. Adherencia a los tratamientos a largo plazo. Pruebas para la acción. Ginebra: OMS; 2004. (citado 19 de abril 2021) Disponible en: URL: http://www. paho.org/Spanish/AD/DPC/NC/adherencia-largo-plazo.pdf

3. Martín L. Acerca del concepto de adherencia terapéutica. Rev Cubana Salud Pública. 2004;(30)4(citado 19 abril 2021) Disponible en: <http://scielo.sld.cu/scielo.php?script=sci_arttext\&pid=S086434662004000400008\&lng=es\&nrm=iso >. ISSN 0864-3466.

4. Devine F, Edwards T, Feldman SR. Barriers to treatment: describing them from a different perspective. Patient Prefer Adherence 2018; 12:129-133. doi:10.2147/PPA.S147420. eCollection 2018. URL: https://doi.org/10.2147/PPA.S147420

5. de Benedictis $D$, Bush A. The challenge of asthma in adolescence. Pediatr Pulmonol 2007;42 (8):683-692. doi: 10.1002/ ppul.20650. URL: https://doi.org/10.1002/ppul.20650

6. Desager K, Vermeulen F, Bodart E. Adherence to asthma treatment in childhood and adolescence - a narrative literature review. Acta Clin Belg. 2018;73(5):348-355. doi: 10.1080/17843286.2017.1409684. Epub 2017 Dec 11. URL: https://doi.org/10.1080/17843286.2017.1409684

7. Klok T, Lubbers S, Kaptein AA, et al. Every parent tells a story: why non- adherence may persist in children receiving guideline-based comprehensive asthma care. J Asthma 2014;51(1):106-112. doi:10.3109/02770903.2013.841191. Epub 2013 Oct 9 URL: https://doi.org/10.3109/02770903.201 3.841191

8. Hughes DA, Bagust A, Haycox A, Walley T. The impact of non-compliance on the cost-effectiveness of pharmaceuticals: a review of the literature. Health Econ. 2001;10(7):601-615. doi: 10.1002/hec.609 URL: https://doi.org/10.1002/hec.609

9. Santer M, Ring N, Yardley L, Geraghty AWA, Wyke S. Treatment nonadherence in pediatric long-term medical conditions: systematic review and synthesis of qualitative studies of caregivers' views. BMC Pediatr 2014; 14:63-72. doi: 10.1186/14712431-14-63. URL: https://doi.org/10.1186/1471-2431-14-63

10. Wijga AH, Zuidgeest MGP, Kerkhof M, Koppelman GH, Smit HA, de Jongste JC. Guideline-recommended use of asthma medication by children is associated with parental information and knowledge: the PIAMA birth cohort. Pharmacoepidemiol Drug Saf 2014. 23(4):406 - 410. doi: 10.1002/pds.3584. URL: https://doi.org/10.1002/pds.3584 Epub 2014 Feb 12.

11. Deis JN, Spiro DM, Jenkins CA, Buckles TL, Arnold DH. Parental knowledge and use of preventive asthma care measures in two pediatric emergency departments. J Asthma 2010;47(5):551-556. doi:10.3109/02770900903560225 URL: https://doi.org/10.3109/02770900903560225

12. Mosnaim G, Li H, Martin M, et al. Factors associated with le- 
vels of adherence to inhaled corticosteroids in minority adolescents with asthma. Ann Allergy Asthma Immunol 2014;112(2):116-120. doi: 10.1016/j.anai.2013.11.021. Epub URL: https://doi.org/10.1016/j.anai.2013.11.021 2013 Dec 21.

13. Chateaux V, Spitz E. Perception de la maladie et adherence therapeutique chez des enfants asthmatiques. Pratiques Psychologiques 2006;12(1):1-16. URL: https://doi.org/10.1016/j. prps.2006.01.001

14. Burgess SW, Sly PD, Morawska A, Devadason SG. Assessing adherence and factors associated with adherence in young children with asthma. Respirology.2008; 13(4):559-563. doi: 10.1111/j.1440-1843.2008.01292. x. URL: https://doi.org/10.1111/j.1440-1843.2008.01292.x Epub 2008 Apr 14.

15. Mulvaney SA, Ho YX, Cala CM, et al. Assessing adolescent asthma symptoms and adherence using mobile phones. J Med Internet Res 2013;15(7): e141. URL: https://doi.org/10.2196/ jmir.2413

16. Blaakman SW, Cohen A, Fagnano M, Halterman JS. Asthma medication adherence among urban teens: a qualitative analysis of barriers, facilitators and experiences with school-based care. J Asthma 2014;51(5):522-529. URL: https://doi.org/10.31 09/02770903.2014.885041 doi:10.3109/02770903.2014.88504 1. Epub 2014 Feb 7.

17. Koster ES, Philbert D, Winters NA, Bouvy ML. Adolescents' inhaled corticosteroid adherence: the importance of treatment perceptions and medication knowledge. J Asthma 2015;52(4):431436. URL: https://doi.org/10.3109/02770903.2014.979366 doi: 10.3109/02770903.2014.979366. Epub 2014 Nov 5

18. Paquet F. Sex, drugs, and rock $\mathrm{n}^{\prime}$ roll: the problems of adolescent and asthma. Paediatr Respir Rev 2006;7:S161-S162. doi: 10.1016/j.prrv.2006.04.215. Epub URL: https://doi.org/10.1016/j.prrv.2006.04.215 2006 Jun 6.

19. Peters TE, Fritz GK. Psychological considerations of the child with asthma. Child Adolesc Psychiatr Clin N Am 2010; 19:319-33. doi:10.1016/j.chc.2010.01.006 URL: https://doi.org/10.1016/j.chc.2010.01.006

20. Bender BG. Risk taking, depression, adherence, and symptom control in adolescents and young adults with asthma. Am J Resipir Crit Care Med 2006; 173:953-7. doi: 10.1164/ rccm.200511-1706PP. Epub 2006 Jan 19. URL: https://doi. org/10.1164/rccm.200511-1706PP

21. Bender B, Zhang L. Negative affect, medication adherence, and asthma control in children. J Allergy Clin Immunol 2008; 122:490-5. URL: https://doi.org/10.1016/j.jaci.2008.05.041 doi:10.1016/j.jaci.2008.05.041. Epub 2008 Jul 7.

22. Bruzzese JM, Fisher PH, Lemp N, Warner CM. Asthma and social anxiety in adolescents. J Pediatr 2009; 155 (3):398-403. doi:10.1016/j.jpeds.2009.04.004. Epub 2009 Jun 24 URL: https://doi.org/10.1016/j.jpeds.2009.04.004

23. Ferro MA, Boyle MH. Self-concept among youth with a chronic illness: a meta- analytic review. Health Psychol 2013;32 (8):839848. doi: 10.1037/a0031861. URL: https://doi.org/10.1037/ a0031861 Epub 2013 Mar 11

24. Kuo CJ, Chen VC, Lee WC, et al. Asthma and suicide mortality inyoung people: a 12-year follow-up study. Am J Psychiatry
2010; 167 (9):1092-1099. URL: https://doi.org/10.1176/appi. ajp.2010.09101455 doi:10.1176/appi.ajp.2010.09101455. Epub $2010 \mathrm{Jul} 15$.

25. Katon WJ, Richardson L, Lozano P, McCauley E. The Relationship of Asthma and anxiety disorders. Psychosom Med. 2004; 66 (3):349-55. URL: https://doi. org/10.1097/01.psy.0000126202.89941.ea doi: 10.1097/01. psy.0000126202.89941.ea. URL: https://doi.org/10.1097/01. psy.0000126202.89941.ea

26. Naimi DR, Freedman TG, Ginsburg KR, Bogen D, Rand CS, Apter AJ. Adolescents and asthma: Why bother with our meds?JACI 2009;123(6):1335- URL: https://doi.org/10.1016/j. jaci.2009.02.0221341. doi: 10.1016/j.jaci.2009.02.022. Epub 2009 Apr 22. URL: https://doi.org/10.1016/j.jaci.2009.02.022

27. Lu Y, Mak K-K, van Bever HPS, Ng TP, Mak A, Ho RC. Prevalence of anxiety and depressive symptoms in adolescents with asthma: A meta-analysis and meta-regression. Pediatric Allergy and Immunology 2012;23 (8):707-15doi: 10.1111/pai.12000 URL: https://doi.org/10.1111/pai.12000

28. Penza-Clyve SM, Mansell C, McQuaid EL. Why don't children take their asthma medications? A qualitative analysis of children's perspectives on adherence. J Asthma 2004;41 (2):18997. doi: 10.1081/jas-120026076. URL: https://doi.org/10.1081/ JAS-120026076

29. Jentzsch N, Camargos P, Colosimo E, Bousquet J. Monitoring adherence to beclomethasone in asthmatic children and adolescents through four different methods. Allergy 2009;64 (10):1458-62. doi: 10.1111/j.1398- 9995 .2009. URL: https:// doi.org/10.1111/j.1398-9995.2009.02037.x doi: 02037.x. Epub 2009 Mar 28.

30. Koster ES, Wijga AH, Koppelman GH, et al. Uncontrolled asthma at age 8: the importance of parental perception towards medication. Pediatric Allergy and Immunology 2011;22 (5):462-8. doi: 10.1111/j.1399-3038.2011.01150.x. Epub URL: https://doi. org/10.1111/j.1399-3038.2011.01150.x 2011 Feb 20.

31. Schatz M, Zeiger RS, Yang SJ, et al. Change in asthma control over time: predictors and outcomes. J Allergy Clin Immunol Pract. 2014;2 (1):59-64. doi:10.1016/j.jaip.2013.07.016. URL: https://doi.org/10.1016/j.jaip.2013.07.016

32. Lindsay JT, Heaney LG. Nonadherence in difficult asthma - facts, myths, and a time to act. Patient Prefer Adherence 2013;7:329336. doi: 10.2147/PPA.S38208. Print 2013

33. Wang LY, Zhong Y, Wheeler L. Direct and Indirect Costs of Asthma in School- age Children. Prev Chronic Dis 2005; 2 (1): A11. Epub 2004 Dec 15. PMID: 15670464; PMCID: PMC1323314.

34. Cohen R, Franco K, Motlow F, Reznik M, Ozuah PO. Perceptions and attitudes of adolescents with asthma. J Asthma 2003; 40:207-11. doi: 10.1081/jas- URL: https://doi.org/10.1081/JAS120017992

35. Rhee H, Wenzel J, Steeves RH. Adolescents' psychosocial experiences living with asthma: a focus group study. J Pediatr Health Care 2007; 21:99-107. doi: 10.1016/j.pedhc.2006.04.005 URL: https://doi.org/10.1016/j.pedhc.2006.04.005

36. Rapoff MA, Lootens CC, Tsai MS. Assessing Adherence and Barriers to Adherence in Pediatric Asthma. Respiratory Drug De- 
livery 2012;1:239-50

37. Bitsko MJ, Everhart RS, Rubin BK. The Adolescent with Asthma. Paediatr Respir Rev 2014;15(2):146-163. doi: 10.1016/j.prrv.2013.07.003. URL: https://doi.org/10.1016/j. prrv.2013.07.003

38. Anderson WC 3rd, Szefler SJ. New and future strategies to improve asthma control in children. J Allergy Clin Immunol 2015;136(4):848-859. URL: https://doi.org/10.1016/j. jaci.2015.07.007 doi: 10.1016/j.jaci.2015.07.007. Epub 2015 Aug 28.

39. Melero Moreno C, Rodríguez Meleán N, Díaz Campos R. Adherencia al tratamiento en asma. Situación actual. Rev Asma 2017;2(1):13-22

40. Ahmad A, Sorensen K. Enabling and hindering factors influencing adherence to asthma treatment among adolescents: $\mathrm{A}$ systematic literature review. J Asthma 2016; 53(8): 1-17. doi: 10.3109/02770903.2016.1155217. URL: https://doi.org/10.310 9/02770903.2016.1155217

41. Lindsay JT, Heaney LG. Non-adherence in difficult asthma and advances in detection. Expert Rev Respir Med. 2013;76:60714. doi: 10.1586/17476348.2013.842129. URL: https://doi.org/ 10.1586/17476348.2013.842129

42. Krishnan JA, Bender BG, Wamboldt FS, et al. Adherence to inhaled corticosteroids: an ancillary study of the childhood asthma management program clinical trial. J Allergy Clin Immunol. 2012;129(1):112-118. doi: 10.1016/j.jaci.2011.10.030. URL: https://doi.org/10.1016/j.jaci.2011.10.030

43. Armstrong ML, Duncan CL, Stokes JO, et al. Association of caregiver health beliefs and parenting stress with medication adherence in preschoolers with asthma. J Asthma 2014;51(4):366-372. doi: 10.3109/02770903.2013.876431 URL: https://doi.org/10.3109/02770903.2013.876431

44. Sumino K, Cabana MD. Medication adherence in asthma patients. Curr Opin Pulm Med 2013;19(1):49-53. doi: 10.1097/ MCP.0b013e32835b117a. URL: https://doi.org/10.1097/MCP. Ob013e32835b117a

45. Weinstein AG. Asthma adherence management for the clinician. J Allergy Clin Immunol Pract 2013;1(2) :123-8. doi: 10.1016/j.jaip.2013.01.009 URL: https://doi.org/10.1016/j. jaip.2013.01.009

46. Plaza V, Fernández-Rodríguez C, Melero C et al.; TAI Study Group. Validation of the 'Test of the Adherence to Inhalers' (TAl) for Asthma and COPD Patients. J Aerosol Med Pulm Drug Deliv.2016;29:142-52. doi: 10.1089/jamp.2015.1212. Epub 2015 Jul 31.

47. Vrijens B, Dima AL, Van Ganse E, et al. What We Mean, When We Talk About Adherence in Respiratory Medicine. J Allergy Clin Immunol Pract 2016;4:802-12. doi: 10.1016/j.jaip.2016.05.019. URL: https://doi.org/10.1016/j.jaip.2016.05.019

48. Apter AJ, Wang X, Bogen DK, et al. Problem solving to improve adherence $\}$ and asthma outcomes in urban adults with moderate or severe asthma: a randomized controlled trial. J Allergy Clin Immunol 2011; 128:516-23doi: 10.1016/j.jaip.2016.05.019. URL: https://doi.org/10.1016/j.jaip.2016.05.019

49. Chan AHY, Reddel HK, Apter A, Eakin M, Riekert K, Foster JM.
Adherence monitoring and e-health: how clinicians and researchers can use technology to promote inhaler adherence for asthma. J Allergy Clin Immunol Pract. 2013;1(5):446-54. doi: 10.1016/j.jaip.2013.06.015. URL: https://doi.org/10.1016/j. jaip.2013.06.015

50. Bender BG. Advancing the science of adherence measurement: implications for the clinician. J Allergy Clin Immunol Pract 2013;1(1):92-3. doi: 10.1016/j.jaip.2012.10.007. URL: https://doi.org/10.1016/j.jaip.2012.10.007

51. D'Arcy S, MacHale E, Seheult J, et al. A method to assess adherence in inhaler use through analysis of acoustic recordings of inhaler. PLoS One. 2014 Jun 6;9(6): e98701. doi: 10.1371/ journal.pone.0098701. eCollection 2014. URL: https://doi. org/10.1371/journal.pone.0098701

52. Holmes MS, Seheult JN, O'Connell P, et al. An acoustic-based method to detect and quantify the effect of exhalation into a dry powder inhaler. J Aerosol Med Pulm Drug Deliv 2015;28 (4):247-53. doi: 10.1089/jamp.2014.1169. URL: https://doi. org/10.1089/jamp.2014.1169

53. Sulaiman I, Seheult J, Sadasivuni N. he Impact of Common Inhaler Errors on Drug Delivery: Investigating Critical Errors with a Dry Powder Inhaler. J Aerosol Med Pulm Drug Deliv. 2017. doi: 10.1089/jamp.2016.1334. Epub 2017 Mar 9. URL: https:// doi.org/10.1089/jamp.2016.1334

54. Sulaiman I, Seheult J, MacHale E, et al. Irregular and Ineffective: A Quantitative Observational Study of the Time and Technique of Inhaler Use. J Allergy Clin Immunol Pract. 2016;4 (5):900-9. doi:10.1016/j.jaip.2016.07.009. URL: https://doi.org/10.1016/j.jaip.2016.07.009

55. Bourdin A, Halimi L, Vachier I, et al. Adherence in severe asthma. Clin Exp Allergy 2012;42 (1):1566-74. doi: 10.1111/j.13652222.2012.04018.x. URL: https://doi.org/10.1111/j.13652222.2012.04018.x

56. Mulhall AM, Zafar MA, Record S, Channell H, Panos RJ. A Tablet-Based Multimedia Education Tool Improves Provider and Subject Knowledge of Inhaler Use Techniques. Respir Care 2016;62 (2):163-71. doi:10.4187/respcare.05008. URL: https:// doi.org/10.4187/respcare.05008

57. Bhogal S, Zemek R, Ducharme FM. Written action plans for asthma in children. Cochrane Database Syst Rev. 2006 Jul 19;(3):CD005306. doi: 10.1002/14651858.CD005306.pub2 URL: https://doi.org/10.1002/14651858.CD005306.pub2

58. Pollard S, Bansback N, FitzGerald JM, Bryan S. The burden of nonadherence among adults with asthma: a role for shared decision-making. Allergy 2017;72 (5):705-12. doi: 10.1111/ all.13090. Epub 2017 Jan 27 URL: https://doi.org/10.1111/ all.13090

59. Braido F, Baiardini I, Sumberesi M, Blasi F, Canonica GW. Obstructive lung diseases and inhaler treatment: results from a national public pragmatic survey. Respir Res 2013;14 (1):94. doi: 10.1186/1465-9921-14-94 URL: https://doi.org/10.1186/14659921-14-94

60. Nickels A, Dimov V. Innovations in technology: social media and mobile technology in the care of adolescents with asthma. Curr Allergy Asthma Rep 2012;12 (6):607-12. doi: 10.1007/s11882- 
012-0299-7 URL: https://doi.org/10.1007/s11882-012-0299-7

61. Price D, Lee AJ, Sims EJ, et al. Characteristics of patients preferring once-daily controller therapy for asthma and COPD: a retrospective cohort study. Prim Care Respir J 2013;22(2):1618. doi: 10.4104/pcrj.2013.00017. URL: https://doi.org/10.4104/ pcrj.2013.00017

62. Ahmed S, Ernst P, Bartlett SJ, et al. The Effectiveness of Web-Based Asthma Self-Management System, My Asthma Portal (MAP): A Pilot Randomized Controlled Trial. J Med Internet Res 2016;18 (12): e313. doi: 10.2196/jmir.5866 URL: https://doi. org/10.2196/jmir.5866

63. Rikkers-Mutsaerts ER, Winters AE, Bakker MJ, et al. Internet-based self-man- agement compared with usual care in adolescents with asthma: a randomized controlled trial. Pediatr Pulmonol 2012;47 (12):1170-9. doi:10.1002/ppul.22575 URL: https://doi.org/10.1002/ppul.22575

64. Seid M, D'Amico EJ, Varni JW, et al. The in vivo adherence intervention for at risk adolescents with asthma: report of a ran- domized pilot trial. J Pediatr Psychol 2012;37 (4):390-403. doi: 10.1093/jpepsy/jsr107 URL: https://doi.org/10.1093/jpepsy/ jsr107

65. Rhee H, Pesis-Katz I, Xing J. Cost benefits of a peer-led asthma self- management program for adolescents. J Asthma 2012 Aug;49(6):606-13. doi: 10.3109/02770903.2012.694540. URL: https://doi.org/10.3109/02770903.2012.694540

66. Riekert KA, Borrelli B, Bilderback A, Rand CS. The development of a motivational interviewing intervention to promote medication adherence among inner-city, African American adolescents with asthma. Patient Educ Couns 2011 Jan;82(1):117-22. doi: 10.1016/j.pec.2010.03.005. Epub 2010 Apr 3. URL: https:// doi.org/10.1016/j.pec.2010.03.005

67. Duncan $\mathrm{CL}$, Hogan MB, Tien KJ, et al. Efficacy of a Parent-Youth Teamwork Intervention to Promote Adherence in Pediatric Asthma. J Pediatr Psychol 2013:38(6):617-28. doi: 10.1093/jpepsy/jss123. Epub 2012 Dec 17. URL: https://doi. org/10.1093/jpepsy/jss123 